\title{
RELAPSING AXILLARY LYMPHANGIOMA IN AN ELDERLY PATIENT: CASE REPORT AND LITERATURE REVIEW
}

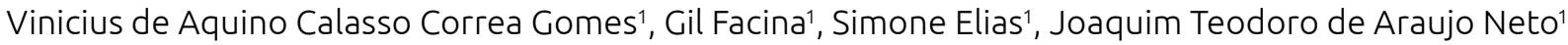 \\ ${ }^{1}$ Universidade Federal de São Paulo, Escola Paulista de Medicina - São Paulo (SP), Brazil.
}

Introduction: Lymphangioma or cystic hygroma is a rare and benign lesion usually found in children or newborns, hardly described after two years of age. It is characterized by congenital obstruction of the lymphatic vessels, which causes dilation and accumulation of lymph inside it. In adults, it can be secondary to trauma and can occur in the neck, head and, more rarely, in the breast and armpit ${ }^{3}$. In this study we present an atypical case of recurrent axillary lymphangioma in an elderly patient. Case report: Female patient, 71 years old, with no history of neoplasms, was referred to the breast surgery center in December 2019 due to bulging in the right axillary region over the past three months, with progressive increase in size. She was submitted to a puncture with complete emptying of the lesion, however, she developed a recurrence of the condition. On initial physical examination, she had bulging in the right axillary region, painless on palpation, softened, measuring about $10.0 \mathrm{~cm}$. Investigation continued with mammography and ultrasound, which did not show any breast changes, but showed a cystic lesion in the right axillary extension of $9.0 \mathrm{~cm}$. The patient returned in March 2020 with recurrence of the condition after another relief puncture, presenting a lesion of about $18 \mathrm{~cm}$, in the same topography and with the same aspect of the previous lesion on physical examination. Initially, it was opted for resection of axillary cyst, however, due to the COVID-19 pandemic, the patient's age and probable benign etiology of the lesion, clinical control was chosen. In August 2020, she presented worsening of the axillary bulging condition, with a lesion measuring about $20.0 \mathrm{~cm}$. We proceeded with the definitive surgical approach with the excision of an axillary cyst with resection of a skin spindle in August 2020. The procedure occurred without complications. The final anatomopathological result showed in cytology the presence of proteinaceous material and mature lymphocytes, corroborating the definitive diagnosis of lymphangioma. During the postoperative period, the patient presented recurrence of the axillary bulging condition, with the need for relief drainage, due to local discomfort. We opted for a new surgical approach to drainage. During the operation, it was possible to observe remnants of the lymphangioma capsule completely adhered to the pectoralis major muscle. We proceeded with vacuum drain and excision of samples from the lesion attached to the muscle was continued. The findings confirmed the diagnosis of lymphangioma. The patient recovered well, with a drain removed on the $10^{\text {th }}$ post operation day. In view of the current situation of the COVID-19 pandemic, it was decided to maintain the clinical follow-up of the patient and she has not had recurrence of the condition so far (five months of follow-up). 